\title{
Ionic-Liquid-Originated Carrier Trapping Dynamics at the Interface in Electric Double-Layer Organic FET
}

\section{Revealed by Operando Interfacial Analyses}

Daijiro Okaue,${ }^{\dagger}$ Ichiro Tanabe, ${ }^{\dagger}$ Sakurako Ono, ${ }^{\dagger}$ Kota Sakamoto,${ }^{\dagger}$ Taiki Sato $^{\dagger}$ Akihito Imanishi, $^{\dagger}$ Yoshitada Morikawa, ${ }^{\ddagger, \S}$ Jun Takeyal and Ken-ichi Fukui ${ }^{*}, \uparrow, \mathbb{\uparrow}$

\footnotetext{
${ }^{\dagger}$ Department of Materials Engineering Science, Graduate School of Engineering Science, Osaka University, Toyonaka, Osaka 560-8531, Japan. E-mail:kfukui@chem.es.osaka-u.ac.jp; Fax:+81-6-6850-6235

${ }^{\ddagger}$ Department of Precision Science and Technology, Graduate School of Engineering, Osaka University, Suita, Osaka 565-0871, Japan.

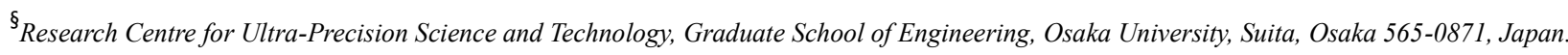

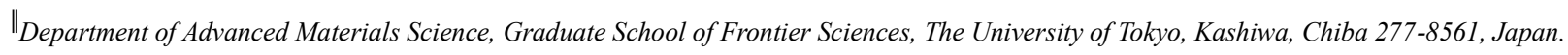

II Institute for Molecular Science (IMS), Okazaki 444-8585, Japan
}

Supporting Note 1: Operando EIS fitting of EDL-OFET with CPE.

Figure S1: Equivalent circuit and operando EI spectra of EDL-OFET.

Table S1: Comparison of device parameters with reported values.

Figure S2: Operando EC-FM-AFM setup and images taken in EMIM-FSI on the rubrene single crystal.

Figure. S3: Frequency shift and force vs distance at $V_{G}=0 \mathrm{~V}$ taken in EMIM-FSI on the rubrene single crystal.

Figure S4: Comparison of densities and self-diffusion coefficients with reported values.

Figure S5: Distributed elementary charge on atoms in the EMIM cation and the FSI anion.

Figure S6: Simulation cell and electrostatic potential profile along the $z$-axis.

Figure S7: Number density profiles of $\mathrm{N}$ atoms in the EMIM cation and the FSI anion.

Figure S8: Construction procedure of a surface distribution (SD) image.

Figure S9: Construction procedure of a time-averaged SD (TASD) image and a differential TASD image.

Figure S10: Differential TASD maps of the second layer.

Figure S11: Conformation distribution of the FSI anion.

Figure S12: Survival probability of ions in the first layer. 


\section{Supporting Note 1: Operando EIS fitting of EDL-OFET with CPE}

The operando EI spectra of bottom-gate bottom-contact EDL-OFET were fitted well with the equivalent circuit in Figure S1a, which represented the distribution of carrier transport in the channel with a constant phase element $\left(\mathrm{CPE}^{1}\right)$ as well as the transmission-line circuit. ${ }^{2-4}$ The impedance of $\mathrm{CPE}\left(Z_{\mathrm{CPE}}\right)$ is represented as

$$
Z_{\mathrm{CPE}}=\frac{1}{(2 \pi \mathrm{j} f)^{p} T}
$$

where $T$ and $p$ are the fitting parameters of the CPE. $f$ is the frequency. The capacitance of CPEEDL is obtained from the imaginary part of $Z_{\mathrm{CPE}}:{ }^{5}$

$$
C_{\mathrm{EDL}}=\frac{T_{\mathrm{EDL}}(2 \pi f)^{p_{\mathrm{EDL}}-1}}{\sin \left(\frac{\pi}{2} p_{\mathrm{EDL}}\right)}
$$

The values of $f$ used in eq. (S2) were $150,150,300$, and $2500 \mathrm{~Hz}$ for $V_{\mathrm{G}}=0.3,0.1,0$, and $-0.2 \mathrm{~V}$, respectively. These values were extracted from the top of the semi-circle in the Cole-Cole plots of the operando EI spectra. The capacitance of the parallel-connected $\mathrm{CPE}_{\mathrm{ch}}$ is obtained by a following simpler equation (without $f$ ): ${ }^{6}$

$$
C_{\mathrm{ch}}=\left(\frac{T_{\mathrm{ch}}}{R_{\mathrm{ch}}^{\left(p_{\mathrm{ch}}-1\right)}}\right)^{1 / p_{\mathrm{ch}}}
$$

The equivalent circuit illustrated in Figure S1a provide channel resistance and EDL capacitance without any approximations in contrast to the transmission-line circuit. The comparison between the fitted results and reported values of rubrene EDL-OFETs with other ILs are summarized in Table. S1. These values were extracted at $V_{\mathrm{G}}=V_{\text {th }}$ - $0.2 \mathrm{~V}$. Note that the fluctuations of the $C-V_{\mathrm{G}}$ and $R-V_{\mathrm{G}}$ curves in the OFF state (Figure 3 in the main text) were owing to the larger fitting error of the operando EI spectra. 

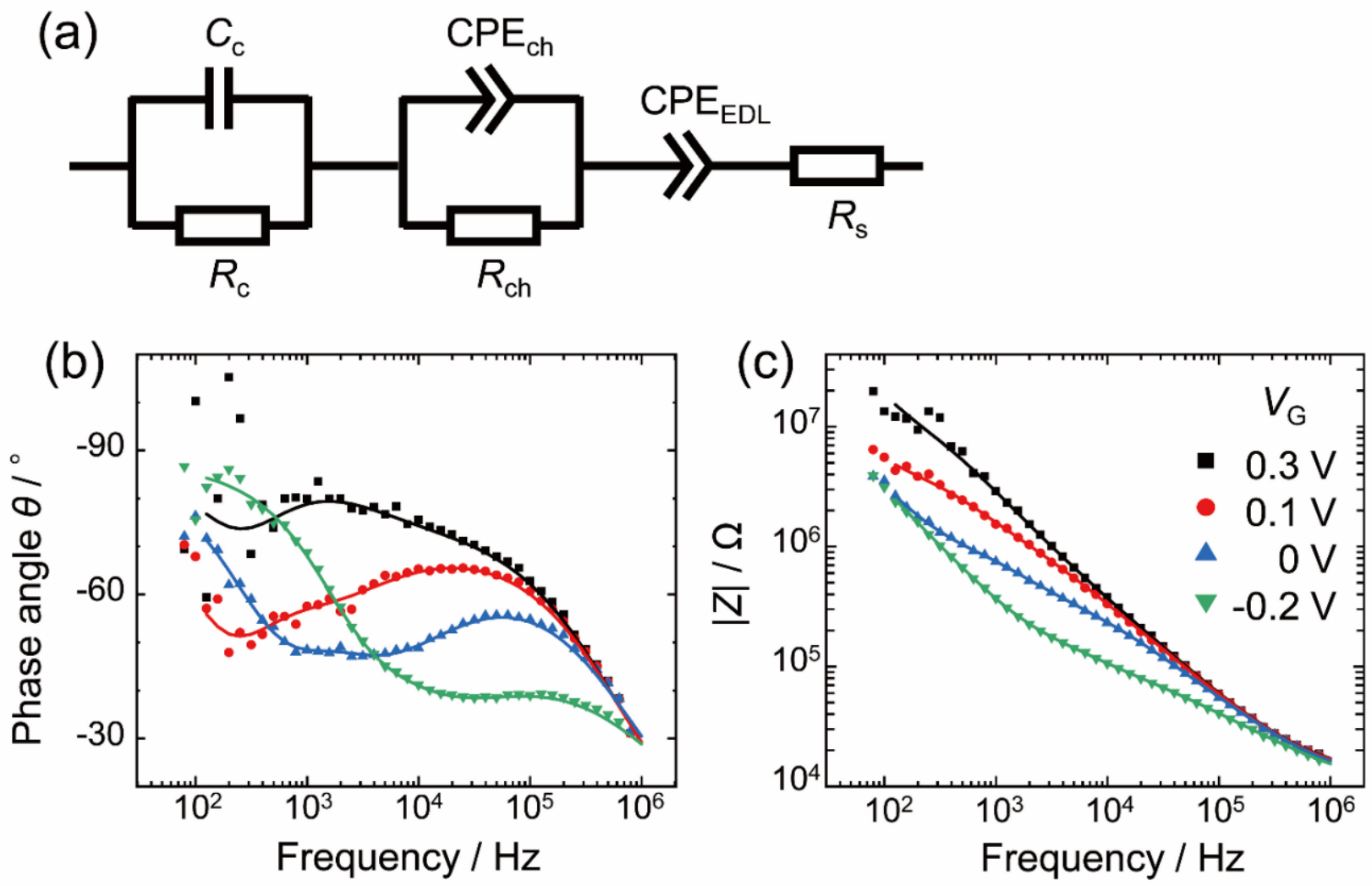

Figure S1. (a) Equivalent circuit between source and gate electrodes of a bottom-gate bottom-contact EDL-OFET. $C_{\mathrm{C}}$ and $R_{\mathrm{C}}$ are the contact capacitance and the resistance between rubrene and the Au (source and drain) electrodes, respectively. $\mathrm{CPE}_{\mathrm{ch}}$ and $R_{\mathrm{ch}}$ are the CPE and resistance of the channel, respectively. CPE $\mathrm{EDL}_{\mathrm{E}}$ is the CPE of EDL between the IL and rubrene interface. $R_{\mathrm{s}}$ is the solution resistance of IL. $(\mathrm{b}, \mathrm{c})$ Operando-EI spectra of EDL-OFET for various $V_{\mathrm{G}}$. (b) Phase angle $\theta$ and (c) $|Z|$ vs. frequency. The dots are the measured results, and the solid lines are their fittings. The fitting range was from $100 \mathrm{~Hz}$ to $1 \mathrm{MHz}$. Black: $V_{\mathrm{G}}=0.3 \mathrm{~V}$ (OFF state), red and blue: $V_{\mathrm{G}}=0.1$ and $0 \mathrm{~V}$, respectively (subthreshold (S.S.) region), and green: $V_{\mathrm{G}}=-0.2 \mathrm{~V}$ (ON state). In the OFF state, $|Z|$ was larger than $10^{7} \Omega$ at $f<100 \mathrm{~Hz}$, resulting in $I_{\mathrm{G}} \sim 1 \mathrm{nA}$. This value was close to the current limit of the bipotentiostat. Therefore, the $\mathrm{S} / \mathrm{N}$ ratio of the operando EI spectrum deteriorated further, resulting in large errors of the $R-V_{\mathrm{G}}$ and $C-V_{\mathrm{G}}$ curves in the OFF state (Figure 3 in the main text).

Table. S1 Comparison of device parameters between this study and reported values.

\begin{tabular}{|c|c|c|c|}
\hline & This study & \multicolumn{2}{|c|}{ Reported values ${ }^{4,7,8}$} \\
\hline$R_{\mathrm{c}} / \Omega$ & $1.8 \times 10^{4}$ & $1.4 \times 10^{4}$ & \\
\hline$R_{\mathrm{ch}} / \Omega$ & $7.0 \times 10^{4}$ & $3.0 \times 10^{5}$ & $8.3 \times 10^{4}$ \\
\hline$C_{\mathrm{EDL}} / \mu \mathrm{F} \mathrm{cm} \mathrm{cm}^{-2}$ at $100 \mathrm{~Hz}$ & 1.34 & 1.45 & \\
\hline
\end{tabular}



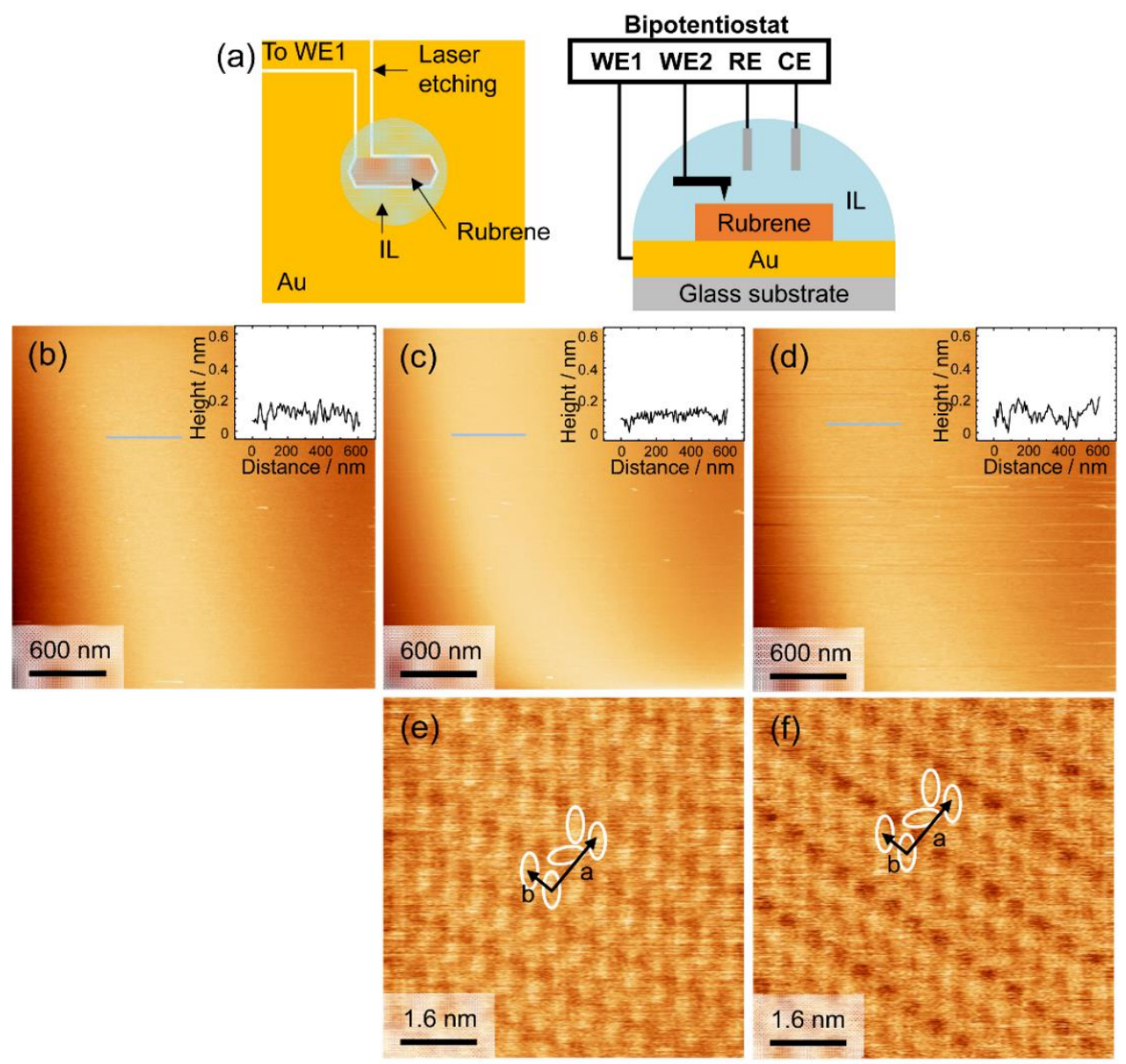

Figure S2. (a) Measurement setup of the operando EC-FM-AFM. The left and right are the top and side views, respectively. (b-f) Operando EC-FM-AFM images taken in EMIM-FSI on the rubrene single crystal. (b) 5 min (c) 40 min and (e) 120 min after carrier injection. (d, f) 5 min after applying $V_{\mathrm{G}}$, detrapping $=1.2 \mathrm{~V}$. (b-d) $3 \times 3 \mu \mathrm{m}^{2}$ images under $V_{\mathrm{G}}=-V_{\mathrm{WE} 1}=-0.3 \mathrm{~V}, V_{\mathrm{WE} 2}=-0.2 \mathrm{~V}, \Delta f=+100 \mathrm{~Hz}, f_{0}=108 \mathrm{kHz}$, and $A_{\mathrm{p}-\mathrm{p}}=0.51 \mathrm{~nm}$. (e) and (f) $8 \times 8 \mathrm{~nm}^{2}$ images under $V_{\mathrm{G}}=-V_{\mathrm{WE} 1}=-0.3 \mathrm{~V}, V_{\mathrm{WE} 2}=-0.2 \mathrm{~V}, \Delta f=+100 \mathrm{~Hz}, f_{0}=109 \mathrm{kHz}$, and $A_{\mathrm{p}-\mathrm{p}}=0.52 \mathrm{~nm}$. The insets of (b-d) are the line profiles at the respective blue lines. The rectangular unit cell of the rubrene single crystal indicated by black arrows in (e) and (f) are 1.45 and $0.72 \mathrm{~nm}$ along the a- and b-axis, respectively, consisting of the reported values. ${ }^{9}$

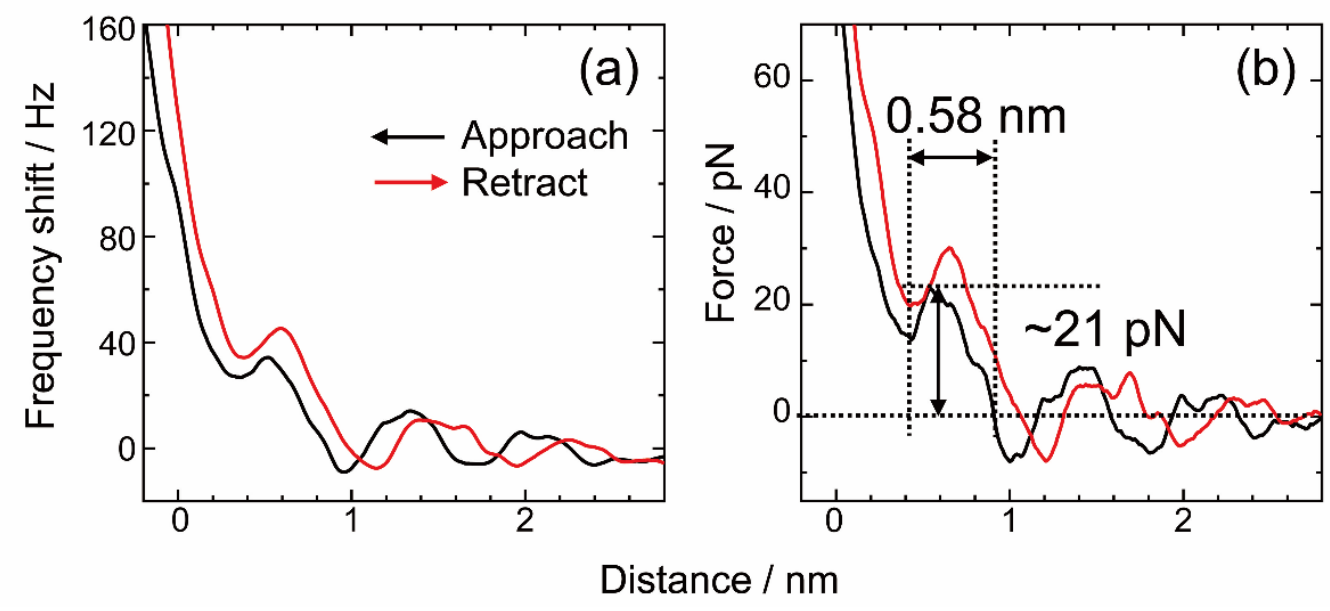

Figure S3. (a) Frequency shift curves as a function of the distance between the cantilever tip and the rubrene surface in EMIM-FSI at $V_{\mathrm{G}}=0 \mathrm{~V}\left(V_{\mathrm{WE} 2}=-0.1 \mathrm{~V}, f_{0}=99 \mathrm{kHz}, A_{\mathrm{p}-\mathrm{p}}=0.42 \mathrm{~nm}\right.$, and approach (retract) speed $\left.=4 \mathrm{~nm} \mathrm{~s}^{-1}\right)$ and (b) force vs distance curves converted from (a) by the method proposed by Sader and Javis ${ }^{10}$. 

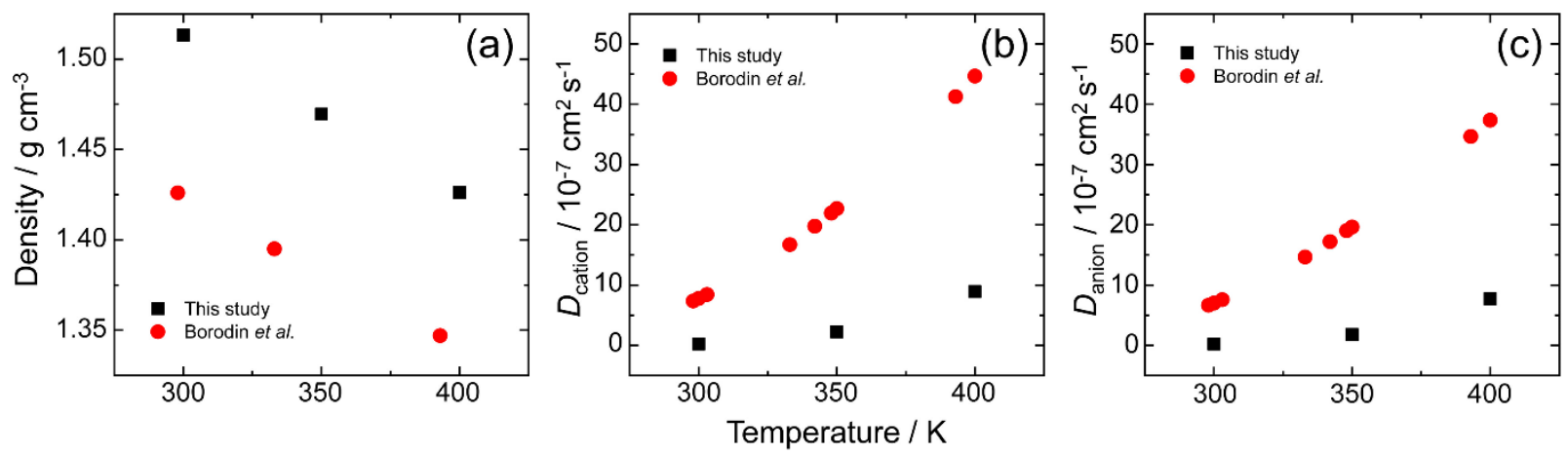

Figure S4. Comparison of densities (a) and self-diffusion coefficients of EMIM cations (b) and FSI anions (c) between our simulations (black squares) and reported results by Borodin et al. (red circles). ${ }^{11}$

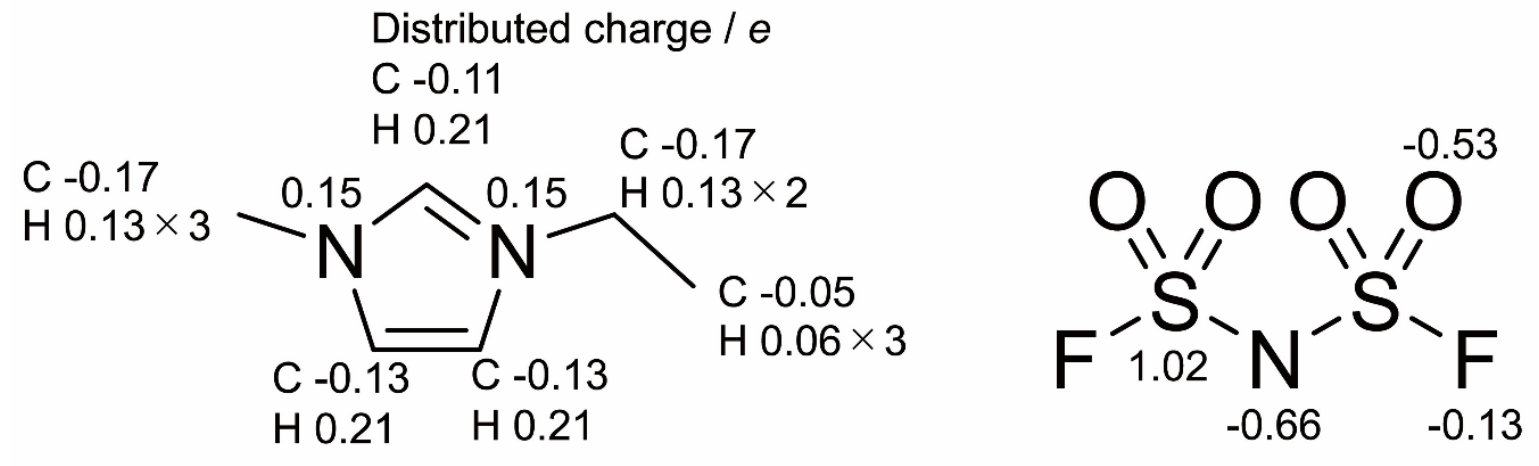

Figure S5. Distributed elementary charge on atoms in the EMIM cation and the FSI anion. ${ }^{12,13}$ 




Figure S6. (a) Simulation cell for an IL/rubrene interface. EMIM cations and FSI anions are represented by red and blue colors, respectively. Non-charged and charged carbon atoms in a rubrene substrate are represented by green and orange colors, respectively. The position of the topmost atoms of the rubrene substrate is defined as $z=0$. (b) Electrostatic potential profiles for whole surface charges estimated by Poisson equation. The charge of the bottomed non-charged rubrene layer was omitted while estimating the potential because its total charge was zero. 

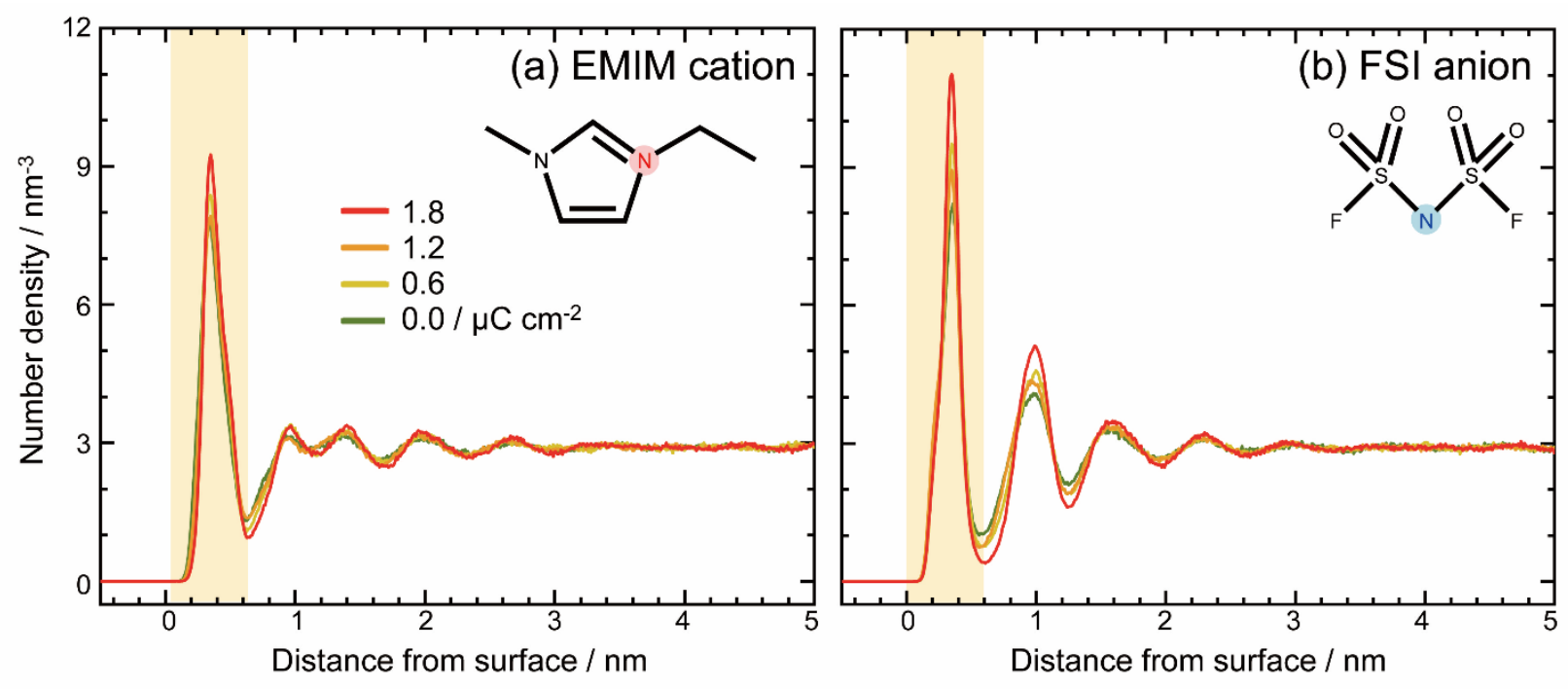

Figure S7. Number density profiles of center $\mathrm{N}$ atoms in (a) the EMIM cation (red-shaded) and (b) the FSI anion (blueshaded) on charged rubrene surfaces. Yellow-shaded areas indicate the first layer $(0<z<0.6 \mathrm{~nm})$. In our previous study of BMIM-TFSI/mica, graphite, ${ }^{14}$ and organic semiconductors interfaces, ${ }^{15}$ we found that the structural and dynamic properties of the cations and anions nearest to the surface $(0<z<0.6 \mathrm{~nm})$ are completely different from those in bulk BMIM-TFSI. In the present study, the region of $0<z<0.6 \mathrm{~nm}$ was defined as the first layer. This value was consistent with the width of the first IL layer observed in operando EC-FM-AFM measurements (Figures 4 and S3). 


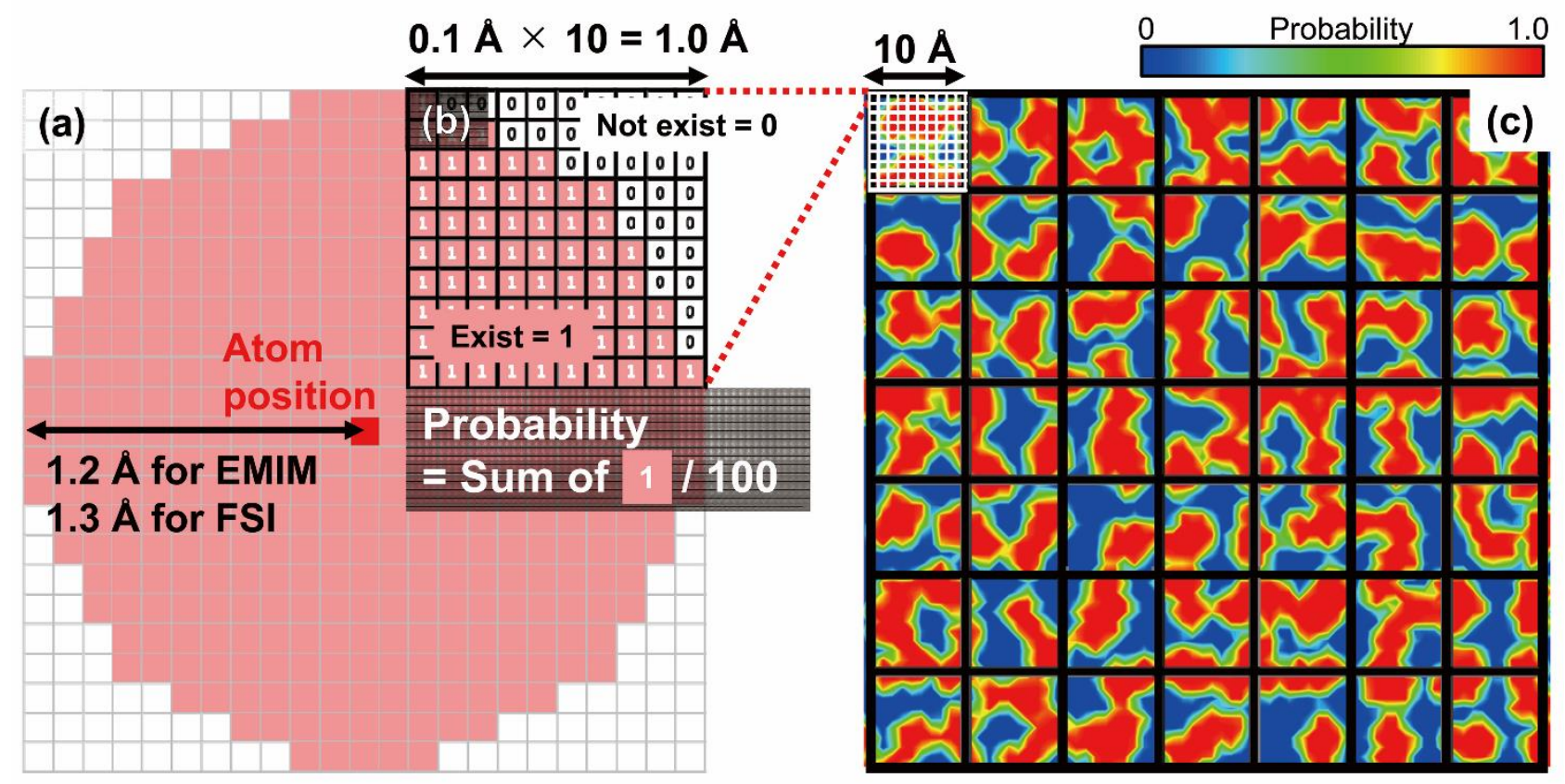

Figure S8. Construction procedure of a surface distribution (SD) image. (a) Individual atoms of the first layer EMIM or FSI ions, whose $z$-positions are less than $6.0 \AA$ from surface, occupy the surface area within 1.2 or $1.3 \AA$, respectively. (b) Occupancy of $0.1 \times 0.1 . \AA^{2}$ areas determined as shown in (a) is digitized as 1 (exist) or 0 (not exist). Probability of the surface occupancy of the matrix $1.0 \times 1.0 \AA^{2}$ area is calculated by summing the stored digital data and divided by 100 . (c) An SD image is constructed as the contour map of the calculated probability values. 
(a)

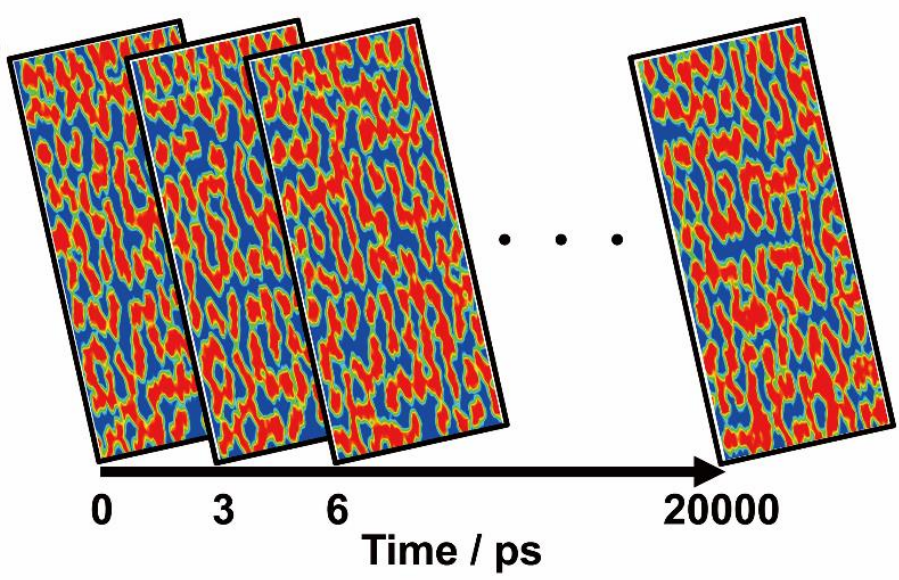

0

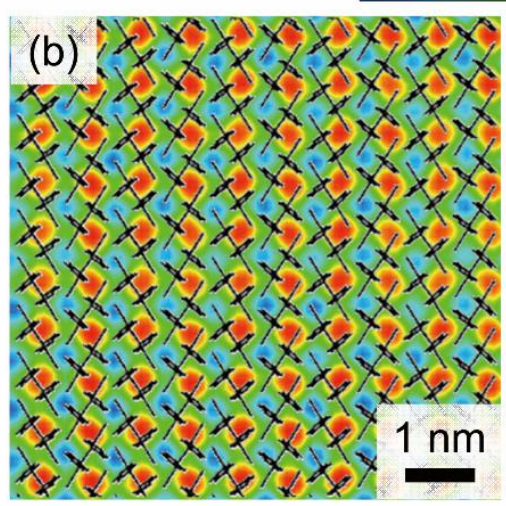

TASD image of EMIM cation
1.0

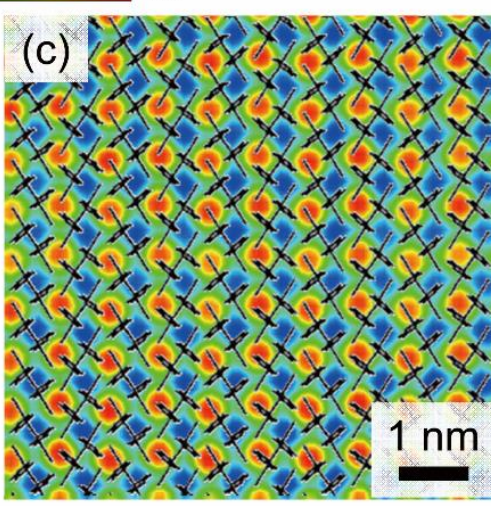

TASD image of FSI anion

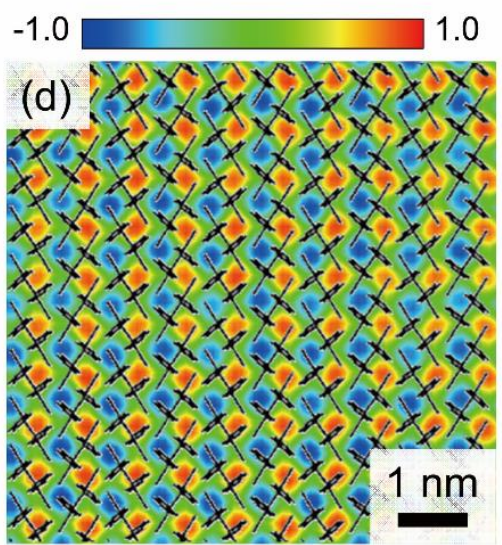

Differential TASD image

Figure S9. Construction procedure of a time-averaged SD (TASD) image and a differential TASD image. (a) Individual SD images are constructed for each 3 ps step for $20 \mathrm{~ns}$ and 6667 SD images are averaged to obtain a TASD image. (b, c) TASD images of (b) EMIM cation and (c) FSI anion, respectively. Averaging decrease the image contrast compared to the SD image shown in Figure S8(c). (d) A differential TASD image as a result of the subtraction of the TASD (FSI) image (b) from the TASD (EMIM) image (c). Thus, red and blue areas indicate the localization of EMIM and FSI, respectively, at the surface for $20 \mathrm{~ns}$. Rubrene molecules in the substrate are overwritten by black solid lines on (b)-(d). Note that the theoretical TASD and differential TASD images of bulk EMIM-FSI, constructed from an infinite simulation, should be monotonic green. 

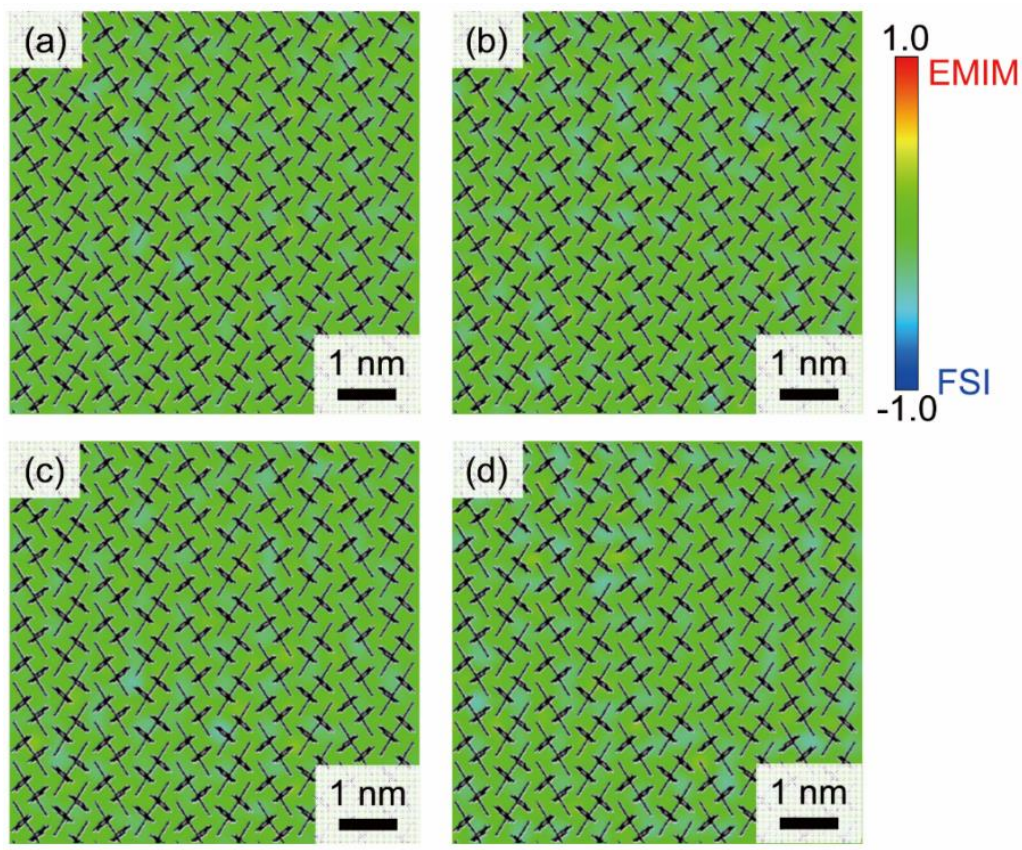

Figure S10. Differential TASD maps $\left(7.05 \times 7.15 \mathrm{~nm}^{2}\right)$ at the second IL layer region $(0.6<z<1.2 \mathrm{~nm})$ constructed from a $20 \mathrm{~ns}$ simulation for the charge density of (a) 0.0 , (b) 0.6 , (c) 1.2 , and (d) $1.8 \mu \mathrm{C} \mathrm{cm}^{-2}$ on the rubrene electrodes. Rubrene molecules in the substrate are overwritten by black solid lines on all figures.
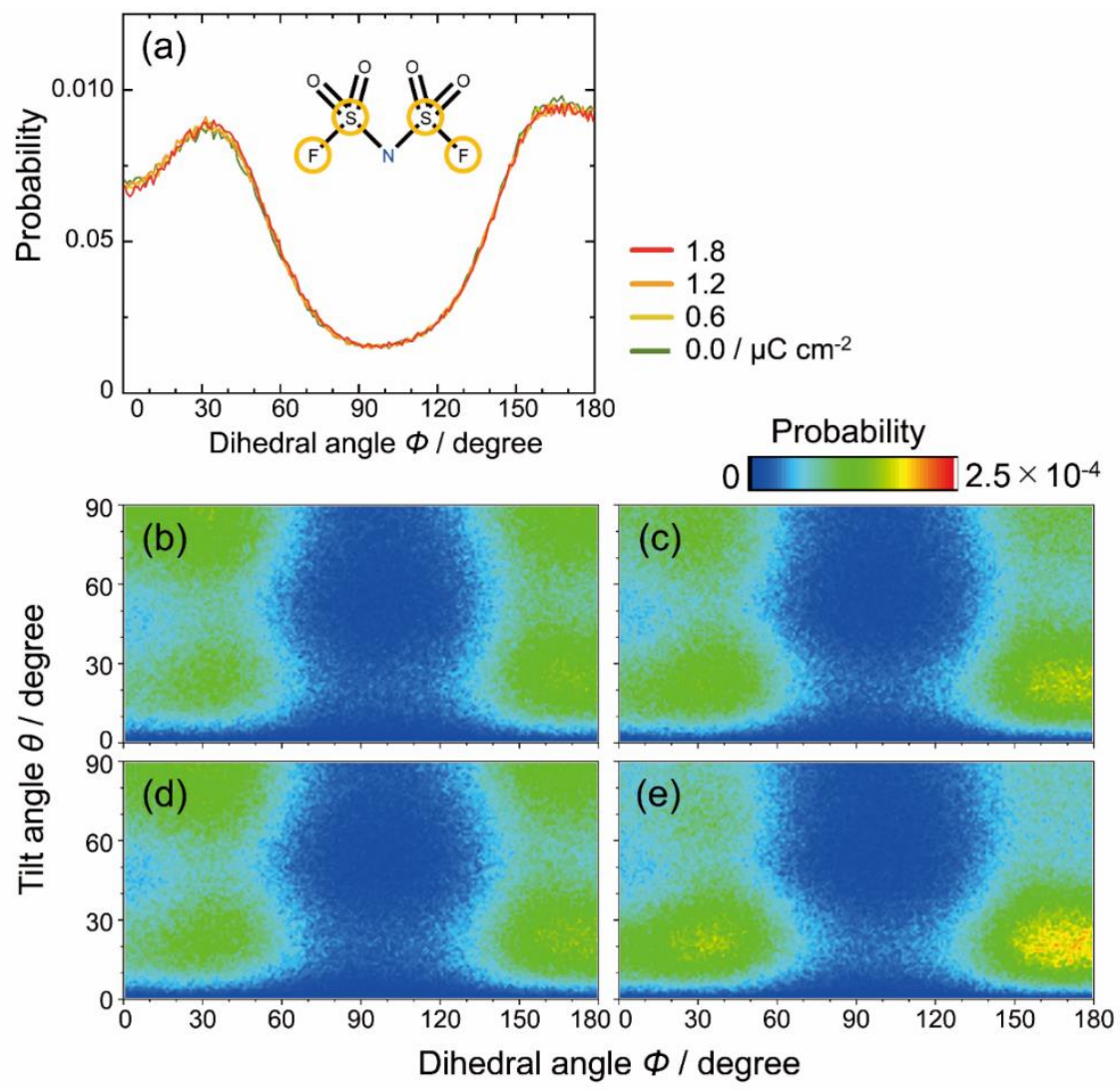

Figure S11. (a) Dihedral angle distribution functions of F-S-S-F in the FSI anions (surrounded by orange circles in the inset) with respect to the injected charge. The degrees of 0 and 180 in horizontal axis suggest cis and trans conformation of the FSI, respectively. (b)-(e) 2D-probability maps of tilt angle of S-S vectors (same as Figure 6c) and dihedral angle of S-F-F-S in the FSI anion at (b) 0.0 , (c) 0.6 , (d) 1.2 , and (e) $1.8 \mu \mathrm{C} \mathrm{cm}^{-2}$. The degrees of 0 and 180 in horizontal axis suggest cis and trans conformation of the FSI. The degree of 0 and 90 in vertical axis suggest standing and lying-flat S-S vectors of FSI anions on the rubrene electrode surface. 


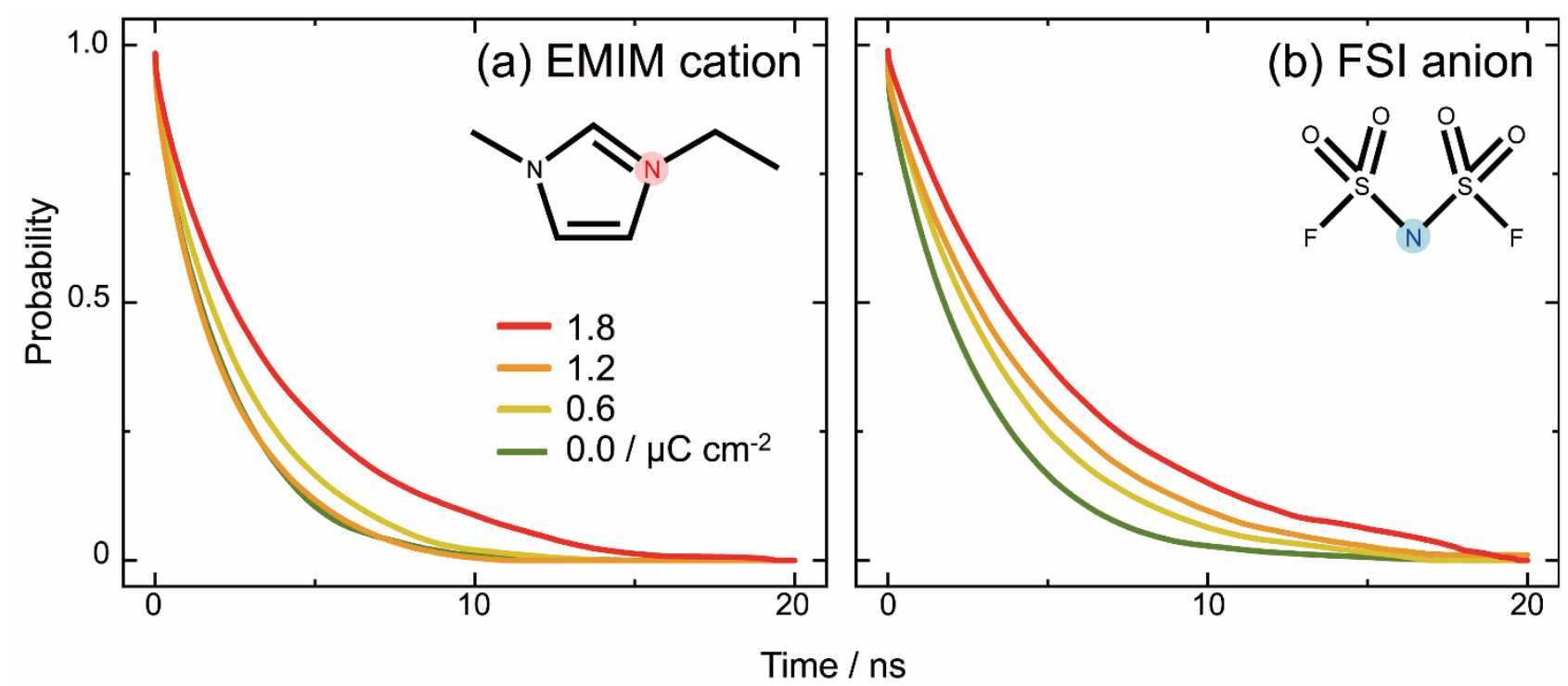

Figure S12. Survival probability of ions in the first layer $(0<z<0.6 \mathrm{~nm})$ facing charged rubrene surfaces for a certain period of time (denoted in the horizontal axis) for (a) EMIM cation and (b) FSI anion. Existence of the ions in the first layer is judged with the $z$ values of the shaded center $\mathrm{N}$ atoms. 


\section{References}

1. Brug, G. J.; Eeden, Van den A. L. G.; Sluyters-Rehbach M.; Sluyters, J. H. The analysis of electrode impedances complicated by the presence of a constant phase element. J. Electroanal. Chem. 1984, 176, 275295.

2. Hamadani, B. H.; Richter, C. A.; Suehle, J. S.; Gundlach, D. J. Insights into the characterization of polymerbased organic thin-film transistors using capacitance-voltage analysis. Appl. Phys. Lett. 2008, 92, 203303.

3. Girolamo, F. V. Di; Barra, M.; Capello, V.; Oronzio, M.; Romano, C.; Cassinese, A. Bias stress instability in organic transistors investigated by ac admittance measurements. J. Appl. Phys. 2010, 107, 114508.

4. Liu, F.; Xie, W.; Shi, S.; Frisbie, C. D.; Ruden, P. P. Coupling of channel conductance and gate-to-channel capacitance in electric double layer transistors. Appl. Phys. Lett. 2013, 103, 193304.

5. Lockett, V.; Sedev, R.; Ralston, J.; Horne, M.; Rodopoulos, T. Differential capacitance of the electrical double layer in imidazolium-based ionic liquids: influence of potential, cation size, and temperature. J. Phys. Chem. C 2008, 112, 7486-7495.

6. Drüschler, M.; Huber, B.; Passerini, S.; Roling, B. Hysteresis effects in the potential-dependent double layer capacitance of room temperature ionic liquids at a polycrystalline platinum interface. J. Phys. Chem. C 2010, $114,3614$.

7. Xia, Y.; Xie, W.; Ruden, P. P.; Frisbie, C. D. Carrier localization on surfaces of organic semiconductors gated with electrolytes. Phys. Rev. Lett. 2010, 105, 036802.

8. Xie, W.; Wang, S.; Zhang, X.; Leighton, C.; Frisbie, C. D. High conductance 2D transport around the Hall mobility peak in electrolyte-gated rubrene crystals Phys. Rev. Lett. 2014, 113, 246602.

9. Minato, T.; Aoki, H.; Fukidome, H.; Wagner, T.; Itaya, K. High-resolution molecular images of rubrene single crystals obtained by frequency modulation atomic force microscopy. Appl. Phys. Lett. 2009, 95, 093302.

10. Sader, J. E.; Javis, S. P. Accurate formulas for interaction force and energy in frequency modulation force spectroscopy. Appl. Phys. Lett. 2004, 84, 1801.

11. Borodin, O.; Gorecki, W.; Smith, G. D.; Armand, M. Molecular dynamics simulation and pulsed-field gradient NMR studies of bis(fluorosulfonyl)imide (FSI) and bis[(trifluoromethyl)sulfonyl]imide (TFSI)-based ionic liquids. J. Phys. Chem. B 2010, 114, 6786-6798.

12. Lopes, J. N. C.; Deschamps, J.; Pádua, A. A. H. Modeling ionic liquids using a systematic all-atom force field. J. Phys. Chem. B 2004, 108, 2038-2047.

13. Shimizu, K.; Almantariotis, D.; Gomes, M. F. C.; Pádua A. A. H.; Lopes, J. N. C. Molecular force field for ionic liquids v: hydroxyethylimidazolium, dimethoxy-2-methylimidazolium, and fluoroalkylimidazolium cations and bis(fluorosulfonyl)amide, perfluoroalkanesulfonylamide, and fluoroalkylfluorophosphate anions. J. Phys. Chem. B 2010, 114, 3592-3600.

14. Yokota, Y.; Miyamoto, H.; Imanishi, A.; Inagaki, K.; Morikawa, Y.; Fukui, K.; Structural and dynamic properties of 1-butyl-3-methylimidazolium bis(trifluoromethanesulfonyl)imide/mica and graphite interfaces revealed by molecular dynamics simulation. Phys. Chem. Chem. Phys. 2018, 20, 6668-6676.

15. Yokota, Y.; Miyamoto, H.; Imanishi, A.; Takeya, J.; Inagaki, K.; Morikawa, Y.; Fukui, K. Microscopic properties of ionic liquid/organic semiconductor interfaces revealed by molecular dynamics simulations. Phys. Chem. Chem. Phys. 2018, 20, 13075-13083.

16. Miyamoto, H.; Yokota, Y.; Imanishi, A.; Inagaki, K.; Morikawa, Y.; Fukui, K. Potential dependent changes in the structural and dynamical properties of 1-butyl-3-methylimidazolium bis(trifluoromethanesulfonyl)imide on graphite electrodes revealed by molecular dynamics Simulations. Phys. Chem. Chem. Phys. 2018, 20, 19408-19415. 